\title{
Blind Adapted, Pre-Whitened Constant Modulus Algorithm
}

\author{
James P. LeBlanc \\ Division of Signal Processing \\ Luleå University of Technology \\ Luleå, Sweden \\ leblancesm.luth.se
}

\author{
Inbar Fijalkow \\ Equipe de Traitement de l'Image et du Signal (ETIS) \\ ENSEA - University of Cergy-Pontoise \\ Cergy, France \\ fijalkow@ensea.fr
}

\begin{abstract}
We examine the use of a blind adaptive "pre-whitening" filter to precede an equalizer adapted by the constant modulus algorithm (CMA). The idea is based on results presented in which the use of a (fixed, or non-adaptive) pre-whitening filter provides an isometry (i.e. geometry preserving transformation) between the combined channel-equalizer (or global space) and the equalizer tap space. As much analysis found in CMA literature is done in "global space" now applies to the pre-whitened "equalizer tap space", considerable exploitation of a known geometric structure is now possible. This paper's main result demonstrates a method for improving the convergence rates of CMA adapted equalizers using the results of prewhitening analysis. The slow convergence of CMA equalizers is a common negative point associated with this well-known, robust blind equalization algorithm.
\end{abstract}

Keywords - Constant Modulus Algorithm (CMA), Blind Equalization, Whitening, Convergence Analysis

\section{INTRODUCTION}

$\mathrm{T}$ HE constant modulus algorithm (CMA) [1], [2], is a wellknown algorithm for blind equalization which has appeared in the recent literature ( [3], [4] contain generous lists of references). Its popularity stems from its implementational simplicity and robustness properties. However, it suffers from slow convergence.

The associated CMA error surface has been analyzed extensively (for example, [5], [6], [7], [8] ). However, much of its analysis in done in the combined "channel-equalizer" (or "global") space. This is often needed to provide understanding of the algorithm that is independent of the actual channel of interest and/or to allow for tractable analysis. However, there are drawbacks to restricting such analysis to the combined channelequalizer space. Specifically, one loses understanding of many convergence issues and transient characteristics of the actual equalizer parameterizations, since it is indeed only the equalizer that is adapted (i.e. not the combined channel-equalizer).

Analysis of the use of a pre-whitening filter on CMA shows that whitening of the received signal that a CMA equalizer with pre-whitened input will have the exact same transient and asymptotic behavior as a system adapted in the combined channel-equalizer space. Such results provide a basis for the use of the adaptive pre-whitener explored herein.

\section{Mathematical Framework}

Consider Figure 1 in which a zero-mean, unit-variance, independent, identically distributed (IID) source sequence $\{s(n)\}$ drawn from a finite alphabet passes through a channel with memory $C(z)$ introducing intersymbol interference (ISI) in the received sequence $\{x(n)\}$. An equalizer $D(z)$ is to be used to remove (or reduce) the ISI. The cascaded channel-equalizer is known as the global response and denote $G(z)$. The transmitted source may then be estimated from the equalizer output sequence $\{y(n)\}$ using a symbol-by-symbol decision device.

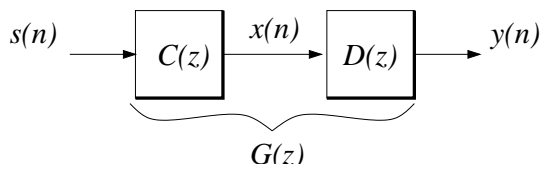

Fig. 1. Simple Channel-Equalizer Block Diagram

Proper equalizer design usually requires knowledge (or estimation) of the channel. Some adaptive methods such as LMS require a bandwidth consuming training sequence (see [9]). Blind equalization [10] allows adaptation to ISI reducing equalizer settings without the need for such training sequences or channel estimates. CMA [2] is a popular blind method which penalizes the deviation of the equalizer output's modulus from some constant $\gamma$. The CMA cost function may be written,

$$
J=\frac{1}{4} \mathrm{E}\left\{\left(|y(n)|^{2}-\gamma\right)^{2}\right\} .
$$

The length $M$ equalizer's tap weights $D(n)$ are adapted using an instantaneous estimate of the gradient of $J$ with respect to 
tap weight vector $D$ via

$$
\begin{aligned}
y(n) & =D^{T}(n) X(n) \\
D(n+1) & =D(n)-\alpha\left(|y(n)|^{2}-\gamma\right) y(n) X^{*}(n)
\end{aligned}
$$

where $X(n)=\left[\begin{array}{llll}x(n) & x(n-1) & \ldots & x(n-(M-1))\end{array}\right]^{T}$ is the M-tap equalizer state vector, $*$ denotes complex conjugation and $\alpha$ is the adaptation stepsize.

\section{KnOwn Results in Global Space}

The CMA error surface is known to be multimodal and contains many saddle points [12], [7], [13]. However, it is also known to exhibit robust performance in a variety of conditions. Due to the nonlinearity of the cost function, the analysis of CMA behavior is difficult and often only possible with some simplifying assumptions.

A common approach is to use an infinite-tap equalizer which means all points in combined channel-equalizer space ( $G$-space) are reachable (assuming an invertible channel $C(z)$ ). In this way, we have an isomorphism (one-to-one and onto relation) between the point in the equalizer space $D$ and points in the combined channel-equalizer space $G$. Using this it has been shown for the noiseless cases [14] that

- CM minima correspond to global transfer function $G(z)=$ $z^{-\nu}$.

- CM saddles are strictly inside the unit sphere.

- CMA (gradient descent adaptation) converges to the minima asymptotically

Additionally, robustness of CMA in additive noise is shown in [17].

Similar methods rely on temporal (or spatial) diversity of fractionally-spaced equalizers (FSE's) (or antenna arrays) to establish this isomorphic relation between the channel-equalizer space and equalizer space. In this way, finite length equalizers may be used also to gain similar results to the infinite-tap equalizers (see [15], [18], [12], [16]).

\section{USE OF WHITENING FILTER}

Next we consider the use of a whitening filter $W(z)$ prior to the equalizer $D_{\mathrm{w}}(z)$ as in Figure 2. It is well-known that for broad class of processes that a causal, stable whitening filter exists [19]. (Note that pre-whitening does not in general correspond to ISI removal.) Such prewhitening may be done as in the algorithm proposed in [11] or in a standard linear prediction method (in an optimal MSE/Weiner Sense). We denote the cascade of the channel and whitening filter as $Q(z)=W(z) C(z)$.

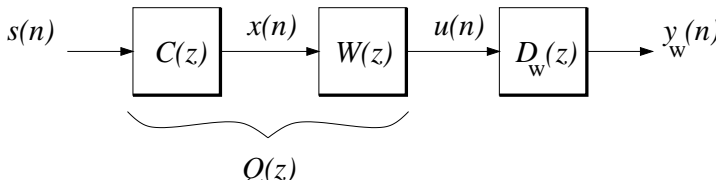

Fig. 2. Pre-whitened Block Diagram

Notice (from Figure 2) that with

$$
\mathcal{Q}=\left[\begin{array}{cccccc}
q_{0} & q_{1} & q_{2} & \ldots & & \\
0 & q_{0} & q_{1} & q_{2} & \ldots & \\
0 & 0 & q_{0} & q_{1} & q_{2} & \ldots \\
& \ddots & \ddots & \ddots & \ddots & \ddots
\end{array}\right]
$$

being the convolution matrix of the cascade channel-whitening filter $Q(z)$ we have several useful relations. The relation between the vectors of source sequence $S$ and vectors of the whitener output $U$ is given by

$$
U=\mathcal{Q}^{H} S
$$

Relating the source autocorrelation matrix to the whitening filter output, we have

$$
\begin{aligned}
\mathcal{R}_{U U} & =\mathrm{E}\left\{U U^{H}\right\} \\
& =\mathrm{E}\left\{\mathcal{Q}^{H} S S^{H} \mathcal{Q}\right\} \\
& =\mathcal{Q}^{H} \mathrm{E}\left\{S S^{H}\right\} \mathcal{Q} \\
& =\mathcal{Q}^{H} \mathcal{R}_{S S} \mathcal{Q}
\end{aligned}
$$

Using the whiteness assumption on the source, and definition of whiteness on $U$, we have $\mathcal{R}_{S S}=\mathcal{R}_{U U}=\mathcal{I}$, and

$$
\mathcal{I}=\mathcal{Q}^{H} \mathcal{Q}
$$

Hence the combined channel-whitening transformation is unitary, that is $\mathcal{Q}^{-1}=\mathcal{Q}^{H}$. A unitary transformation from one space to another has all eigenvalues equal to one and is wellknown to preserve geometries between the two spaces. In other words, it provides an isometry between the two spaces in which distances between points are preserved under the transformation. To see this, use the property of the adjoint being the unique operator which commutes across the inner product operation and consider the distance between two whitening filter output sequences $u_{1}$ and $u_{2}$ when $U=\mathcal{Q}^{H} S$

$$
\begin{aligned}
\left\|u_{1}-u_{2}\right\|^{2} & =\left\langle\left(u_{1}-u_{2}\right),\left(u_{1}-u_{2}\right)\right\rangle \\
& =\left\langle\mathcal{Q}^{H}\left(s_{1}-s_{2}\right), \mathcal{Q}^{H}\left(s_{1}-s_{2}\right)\right\rangle \\
& =\left\langle\left(s_{1}-s_{2}\right), \mathcal{Q}^{H}\left(s_{1}-s_{2}\right)\right\rangle \\
& =\left\|s_{1}-s_{2}\right\|^{2} .
\end{aligned}
$$


In the non-unitary cases, the distance distortion appears in the $\mathcal{R}_{U U}$ term.

We now explore the relationship between the CMA cost function parameterized by the global-space, $J(G)$, and the CMA cost function parameterized by the equalizer in the prewhitened case $J\left(D_{\mathrm{w}}\right)$. For the global space, with $|y|^{2}=$ $\left(G^{H} S\right)\left(G^{H} S\right)^{H}=G^{H} S S^{H} G$ where $S$ is the state vector of the global space and $G$ the global impulse response vector, we get,

$$
\begin{aligned}
J(G) & =\frac{1}{4} \mathrm{E}\left\{\left(|y(n)|^{2}-\gamma\right)^{2}\right\} \\
& =\frac{1}{4} \mathrm{E}\left\{\left(G^{H} S S^{H} G-\gamma\right)^{2}\right\} \\
& =\frac{1}{4}\left(\mathrm{E}\left\{G^{H} S S^{H} G G^{H} S S^{H} G\right\}-2 \gamma G^{H} \mathrm{E}\left\{S S^{H}\right\} G+\gamma^{2}\right) \\
& =\frac{1}{4}\left(G^{H} \mathrm{E}\left\{S S^{H} G G^{H} S S^{H}\right\} G-2 \gamma G^{H} G+\gamma^{2}\right) .
\end{aligned}
$$

The mean gradient of this cost function with respect to $G$ determines the trajectory of a CMA equalizer for the special case when the global parameterization (combined channel-equalizer space) is adjustable is given by

$$
\begin{aligned}
\frac{\partial J(G)}{\partial G} & =\frac{\frac{1}{4} \partial \mathrm{E}\left\{\left(G^{H} S S^{H} G-\gamma\right)^{2}\right\}}{\partial G} \\
& =\mathrm{E}\left\{G^{H} S S^{H} G S S^{H} G\right\}-\gamma \mathrm{E}\left\{S S^{H}\right\} G \\
& =\mathrm{E}\left\{G^{H} S S^{H} G S S^{H} G\right\}-\gamma G .
\end{aligned}
$$

Now consider the prewhitened case with $y_{\mathrm{w}}=D_{\mathrm{w}}^{H} U=$ $U^{H} D_{\mathrm{w}}$ where $U$ is the state vector of the equalizer. Similar steps obtain

$$
\begin{aligned}
J\left(D_{\mathrm{w}}\right)= & \frac{1}{4}\left(D_{\mathrm{w}}^{H} \mathrm{E}\left\{U U^{H} D_{\mathrm{w}} D_{\mathrm{w}}^{H} U U^{H}\right\} D_{\mathrm{w}}\right. \\
& \left.-2 \gamma D_{\mathrm{w}}^{H} D_{\mathrm{w}}+\gamma^{2}\right)
\end{aligned}
$$

Similarly, the mean gradient of this cost function with respect to $D_{\mathrm{w}}$ (the equalizer taps) determines the actual trajectory of a pre-whitened CMA equalizer. Since $U=\mathcal{Q}^{H} S$, the CMA cost function mean gradient for pre-whitened CMA $\left(\frac{\partial J\left(D_{\mathrm{w}}\right)}{\partial D_{\mathrm{w}}}\right)$ can be written in terms of the global system gradient $\frac{\partial J(G)}{\partial G}$ as follows,

$$
\frac{\partial J\left(D_{\mathrm{w}}\right)}{\partial D_{\mathrm{w}}}=\mathcal{Q}^{H} \frac{\partial J(G)}{\partial G} .
$$

Accordingly, the Hessian with respect to $G$ and $D_{\mathrm{w}}$ are related through:

$$
\mathcal{H}_{D_{\mathrm{w}}}\left(D_{\mathrm{w}}\right)=\mathcal{Q}^{H} \mathcal{H}_{G}(G) \mathcal{Q}
$$

where the $i, j$ entry of $\mathcal{H}_{G}(G)$ is given by $\frac{\partial^{2} J(G)}{\partial G_{i} \partial G_{j}}$.

So, in fact, the gradients and Hessians of the CMA cost function between the globally adaptable system and pre-whitened CMA agree up to a unitary transformation. In other words, the behavior of prewhitened CMA is "equivalent to" CMA adapted in the global space (modulo a unitary transformation). The effect of this unitary transformation doesn't affect asymptotic convergence but may affect the convergence speed (compared to the non-prewhitened case). This is similar to the effect of reducing eigenvalue disparity in the filter input autocorrelation matrix in standard LMS. High eigenvalue disparity introduces a "slow" mode which adversely affects convergence speed.

Moreover, we are looking for $D_{\mathrm{w}}$ solving for $\mathcal{Q} D_{\mathrm{w}}=G$ with $G$ having one non-zero entry. Therefore, $D_{\mathrm{w}}=\mathcal{Q}^{H} G$ should belong to the unit sphere. This may lead us to a better initialization of $D_{\mathrm{w}}$ for the CMA adaptation and to eventual modifications of the CMA.

\section{AdAPtive Pre-Whitening}

The above analysis considered a fixed, optimal prewhitened (in the Weiner sense) prewhitening filter. Next we introduce a blind adaptive pre-whitening filter using the robust least mean squares (LMS) algorithm in a linear prediction configuration prior to the CMA adapted equalizer as shown in Figure 3

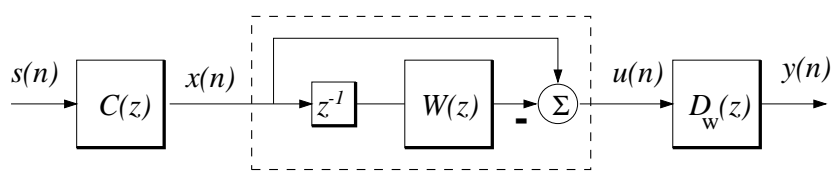

Fig. 3. Adaptive Pre-whitening with Linear Prediction

In this configuration the whitening filter is an LMS linear predictor. It is the error signal (or innovations) that is fed on to the CMA equalizer. The linear predictor's $N$-tap weights are adjusted using LMS as

$$
\begin{aligned}
u(n) & =x(n)-W^{T}(n) \tilde{X}(n) \\
W(n+1) & =W(n)-\beta_{\operatorname{lp}} u(n) \tilde{X}(n)
\end{aligned}
$$

where $\tilde{X}(n)=\left[\begin{array}{llll}x(n-1) & x(n-2) & \ldots & x(n-N)\end{array}\right]^{T}$. The adaptation stepsize parameter for this linear predictor is denoted $\beta_{\mathrm{lp}}$.

The equalizer's $M$ tap weights are adjusted using CMA in the usual fashion.

$$
\begin{aligned}
y(n) & =D_{W}^{T}(n) U(n) \\
D_{W}(n+1) & =D_{W}(n)-\beta_{\text {cma }}\left(|y(n)|^{2}-\gamma\right) y(n) U^{*}(n)
\end{aligned}
$$

where $U(n)=\left[\begin{array}{llll}u(n) & u(n-1) & \ldots & u(n-(M-1)\end{array}\right]^{T}$ is the M-tap equalizer state vector. The stepsize parameter for this CMA equalizer portion of the prewhitened CMA system is denoted $\beta_{\mathrm{cma}}$. 


\section{EXAMPLE}

The following computer simulation demonstrates the performance advantages that the adaptive pre-whitening the input to a standard CMA equalizer provides. In this case a comparison is made with a standard CMA equalizer (denoted CMA) and an adaptive (linear prediction) prewhitened CMA equalizer (denoted LP-CMA) as well as an optimal pre-whitened CMA (denoted WCMA). The channel used is somewhat arbitrary, but deliberately chosen to have a large range of frequency response (i.e. a case wherein CMA is known to have long convergence times). It is interesting to note, that LMS adapted filters also would exhibit a "slow mode" convergence in such cases. However, the quadratic nature of the LMS algorithm appears to be less sensitive to the eigenvalue spread than the CMA's quartic error surface.

The channel taps $C$ are

$$
C=\left[\begin{array}{llllll}
.0003 & .0029 & .0090 & -.0440 & .0468 & -.0485 \\
& .3603 & -.7401 & .5451 & -.1326 & .0350
\end{array}\right]^{T}
$$

which has frequency response as in Figure 4.

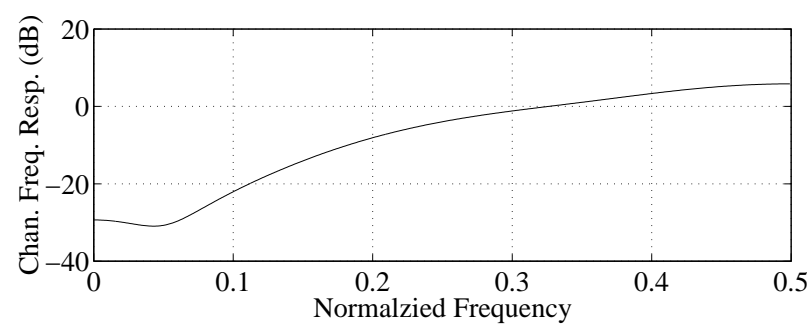

Fig. 4. Channel Frequency Response

The source sequence is iid BPSK with $s(n) \in\{-1,+1\}$, and the CMA dispersion constant is thus $\gamma=1$. The $M(=25)$ initial linear predictor weights and the initial $N(=25) \mathrm{CMA}$ equalizer weights for the all examples (CMA and LP-CMA) are all zero, except for the center tap which equals 1 (using the "standard" center tap initialization).

The adaptation stepsize for the three experiments are shown in Table I. Note that the standard CMA is given a stepsize twice as large as the LP-CMA combination and the WCMA case. This is to allow fairer comparison since the LP-CMA combination enjoys two adaptations (one for the linear predictor, and one for the CMA equalizer) per sample and the "omniscient" WCMA, while standard CMA has only one. Doubling standard CMA's adaptation stepsize is meant to level the playing field. (Although note that the LP-CMA system has more parameters to adjust. Typically adaptive systems with more parameters will take longer time to converge than systems with less parameters.)
TABLE I

STEPSIZE PARAMETERS FOR EXPERIMENTS

\begin{tabular}{l|lr} 
Experiment Name & LP Stepsize & CMA Stepsize \\
\hline CMA & & $\alpha=0.02$ \\
\hline \hline LP-CMA I & $\beta_{\mathrm{lp}}=0.005$ & $\beta_{\text {cma }}=0.01$ \\
LP-CMA II & $\beta_{\mathrm{lp}}=0.01$ & $\beta_{\text {cma }}=0.01$ \\
LP-CMA III & $\beta_{\mathrm{lp}}=0.05$ & $\beta_{\text {cma }}=0.01$ \\
\hline WCMA & & $\alpha=0.01$ \\
\hline
\end{tabular}

Also, in order to allow for proper comparison of the systems with these simulation the adaptation equations used a normalized form (á la normalized LMS - NLMS) in which the adaptation stepsizes are normalized by the filter's regressor's power. Specifically,

- in (3) replace $\alpha$ with $\alpha / X^{H} X$

- in (13) replace $\beta_{\mathrm{lp}}$ with $\beta_{\mathrm{lp}} / \tilde{X}^{H} \tilde{X}$

- in (15) replace $\beta_{\mathrm{cma}}$ with $\beta_{\mathrm{cma}} / U^{H} U$.

Although certainly not necessary for actual implementation, it was used here since the CMA filter's ( $D_{W}$ input power) in the LP-CMA system input power may be fluctuating due to the adapting linear predictor. The normalized adaptation algorithm versions were used in the simulations here for fairer comparison. But it is suspected that prewhitening the CMA input would actually allow a larger adaptation stepsize to be used. This would allow for even faster convergence for the prewhitened CMA case.

Figure 5 shows the equalizer outputs of the two systems. Notice that the prewhitened CMA equalizer achieves faster eyeopening. For a better view of the convergence behavior of these two systems, define a measure of the intersymbol interference (ISI) as

$$
\mathrm{ISI}=\frac{\sum_{i} h_{i}^{2}-\max _{i}\left(h_{i}^{2}\right)}{\max _{i}\left(h_{i}^{2}\right)} .
$$

Figure 6 shows the ISI trajectories (in $\mathrm{dB}$ ) during adaptation for the two systems. Notice that large differences in the slopes on the log scale between the two systems. Also note that the difference between the slopes is increasing: the standard CMA system is "decelerating" while the LP-CMA system maintains the same exponential-constant of convergence.

\section{CONCLUSION}

This work introduces an exploit of the analysis of the prewhitened CMA error surface. Such prewhitening allows the adapted equalizer to traverse a trajectory similar to the trajectory 

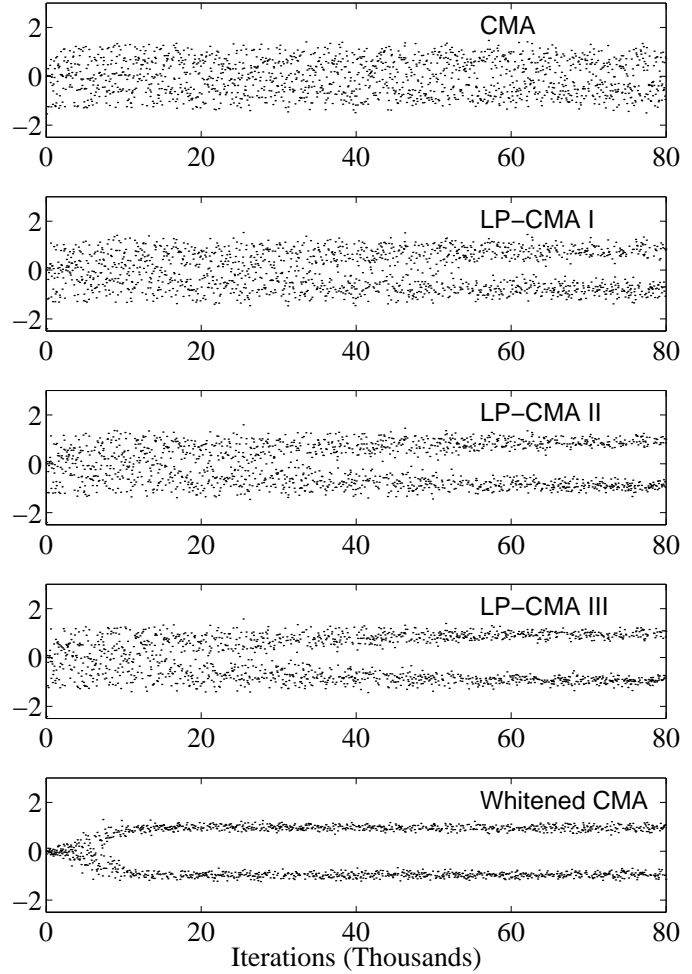

Fig. 5. Equalizer Outputs

which a non-realizable globally (channel-equalizer) adapted system. The geometries of such global channel-equalizer spaces are well understood from the wealth of existing research on CMA. The immediate impact of this allows reduction of "slow" modes of convergence of the CMA equalizer through the use of prewhitening. Specifically, the use of a LMS adaptive filter in a linear predictor configuration is introduced to prewhiten the input to a conventional CMA equalizer. The convergence speed is seen to be markedly improved for channels which exhibits a large frequency response deviation.

\section{REFERENCES}

[1] D.N. Godard, "Self-Recovering Equalization and Carrier Tracking in TwoDimensional Data Communication Systems," IEEE Trans. on Commun., vol. COM-28, No. 11, pp. 1867-75, Nov. 1980.

[2] J.R. Treichler, M.G. Agee, "A New Approach to Multipath Correction of Constant Modulus Signals," IEEE Trans. on Acoustics, Speech, and Signal Processing, vol. ASSP-31, no. 2 pp. 459-72, April 1983.

[3] C. R. Johnson, Jr., P. Schniter, T.J. Enders, J.D. Behm, D.R. Brown, R.A. Casas, "Blind equalization using the constant modulus criterion: A review," Proceedings of the IEEE special issue on Blind System Identification and Estimation, vol. 86, no. 10, pp. 1987-50, Oct. 1998.

[4] S. Haykin, ed., Unsupervised Adaptive Filtering, New York: Wiley, 1999.

[5] I. Fijalkow, C. E. Manlove, C. R. Johnson, Jr., "Adaptive Fractionally Spaced Blind CMA Equalization: Excess MSE," IEEE Transactions on Signal Processing, vol. 46, no. 1, pp. 227-31, Jan. 1998.

[6] H.H. Zeng, L. Tong, C.R. Johnson, Jr., "Relationships between the con-

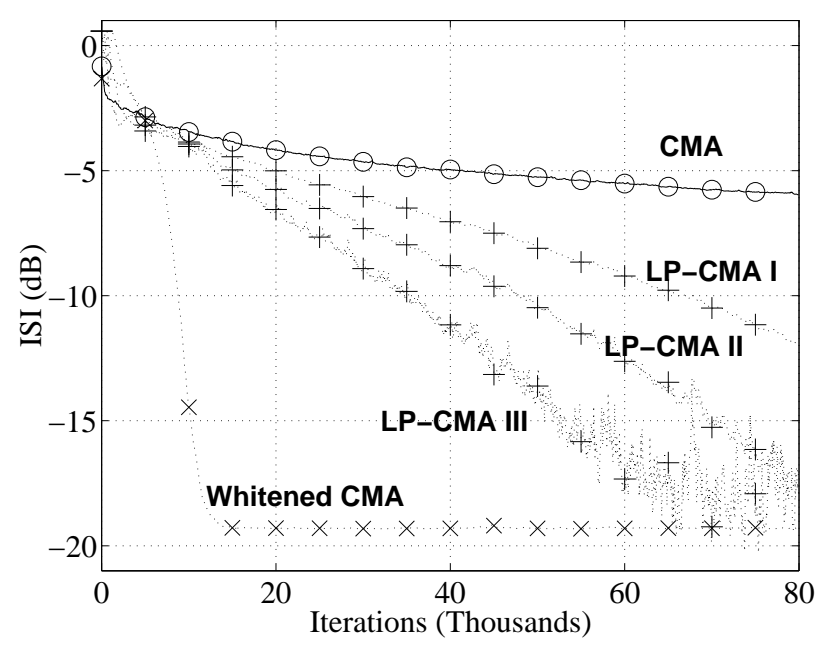

Fig. 6. ISI Trajectory Comparison

stant modulus and Weiner receivers," IEEE Transactions on Information Theory, vol. 44, no. 4, pp. 1523-38, July 1998.

[7] C. R. Johnson, Jr., B. D. O. Anderson, "Godard Blind Equalizer Error Surface Characteristics: White, zero-mean, binary case," Int'l Journal of Adaptive Control and Signal Processing, vol. 9, pp. 301-24, July-Aug, 1995.

[8] S. Lambotharan, J. Chambers, C.R. Johnson, Jr, "Attraction of saddles and slow convergence of CMA adaptation," Signal Processing, vol. 59, no. 2, pp. 335-40, June 1997.

[9] S.U.H. Qureshi, "Adaptive Equalization," Proc. of the IEEE, vol. 73, no. 9, pp. 1349-87, Sept 1985.

[10] S. Haykin, Blind Deconvolution, Englewood Cliff, NJ: Prentice-Hall, 1994.

[11] O. Macchi, "L'Égalisation Numérique en Communications," Ann. Télécommun., 53, no. 1-2, pp. 39-58, 1998.

[12] J.P. LeBlanc, I. Fijalkow, C.R. Johnson, Jr., "CMA fractionally-spaced equalizers: Stationary points and stability under IIE and temporally correlated sources," Int'l Journal of Adaptive Control and Signal Processing, vol. 12, no. 2, pp. 2120-9, Mar. 1998.

[13] A. Benveniste, M. Goursat, G. Ruget, "Robust Identification of a Nonminimum Phase System: Blind Adjustment of a Linear Equalizer in Data Communications," IEEE Trans. on Automat. Contr, vol. AC-25, no. 3, pp. 385-399, Jun. 1980.

[14] G. J. Foschini, "Equalizing Without Altering or Detecting Data," $A T \& T$ Technical Journal, vol. 64, no. 8, pp. 1885-1911, Oct. 1985.

[15] I. Fijalkow, F. Lopez de Victoria, C.R. Johnson, Jr., "Adaptive fractionallyspaced blind equalization," Proc. IEEE Signal Processing Workshop (Yosemite National Park, CA), pp. 257-60, 2-5 Oct. 1994.

[16] W. Chung, J.P. LeBlanc, "The local minima of fractionally-spaced CMA blind equalizer cost function in the presence of channel noise," Proc. IEEE International Conference on Acoustics, Speech, and Signal Processing (Seattle, WA), pp. 3345-8, 12-15 May 1998.

[17] I. Fijalkow, A. Touzni, J.R. Treichler, "Fractionally spaced equalization using CMA: Robustness to channel noise and lack of disparity," IEEE Transactions on Signal Processing, vol. 45, no. 1, pp. 56-66, Jan. 1997.

[18] Y. Li, Z. Ding, "Global Convergence of Fractionally Spaced Godard Adaptive Equalizers," IEEE Transactions on Signal Processing, vol. 44, no. 4, pp. 818-26, Apr. 1996.

[19] G.D.Forney, "Maximum-Likelihood Sequence Estimation of Digital Sequences in the Presence of Intersymbol Interference," IEEE Trans. on Information Theory, vol. IT-18, no. 3, pp. 363-378, May 1972. 